\title{
Familiarity effects and word unitization in visual comparison tasks
}

\author{
HARVEY H. C. MARMUREK \\ University of Guelph, Guelph, Ontario, Canada
}

\begin{abstract}
The decision that two words are identical is made more quickly than the decision that two nonwords are identical. This familiarity effect was shown to be larger in a simultaneous matching task than in a sequential matching task. In the simultaneous task, two words were not matched as quickly as a single letter and a letter in a predesignated location within a word. The latter finding rules out a perceptual unitization account of the familiarity effect (Silverman, 1985). The familiarity effect was interpreted to be due to the facilitated encoding of a comparison item when a holistic cognitive unit representing the target is activated in memory.
\end{abstract}

Although it is generally recognized that the cultural familiarity of a linguistic display affects visual information processing, the specific processes that underlie familiarity effects are not yet fully understood (Henderson, 1982, chap. 9; Krueger, 1975; McClelland \& Mozer, 1986). The present experiments were designed to test the hypothesis that familiarity effects arise because units of different sizes are used in the processing of familiar and unfamiliar displays. The critical assumption is that, given items containing a constant number of letters, there are units available in memory for processing familiar items that are larger than those available for processing unfamiliar items. The rationale leading to the experiments was that familiarity effects would occur only when the different-sized units available in memory were activated in the task given to subjects.

Two research paradigms typically are used in investigations of visual processing units. The paradigms differ in whether the displays are presented under data-limited or resource-limited conditions, and in whether the critical dependent measure is accuracy or latency. Santee and Egeth (1982) have argued that these paradigms tap different aspects of visual information processing, and the results obtained in the two paradigms seem to conflict in their implications about the units used in visual word processing. Under data-limited conditions, decisions about a letter in a word are more accurate than are decisions about an isolated letter (see Henderson, 1982, chap. 9, for a review of the word-superiority effect). Under resource-limited conditions, decisions about a letter in a word are made more slowly than decisions about a letter in isolation, when the items are presented sequentially

This research was supported by Natural Sciences and Engineering Research Council of Canada Grant OGP0000684. The author thanks Ward Pangborn for his assistance in data collection, and R. W. Proctor and two anonymous reviewers for their helpful comments on an earlier version of this paper. Requests for reprints should be addressed to Harvey H. C. Marmurek, Department of Psychology, University of Guelph, Guelph, Ontario N1G 2W1, Canada.
(Johnson, 1975), and there is no difference when the displays are presented simultaneously (Marmurek, 1977).

In contrast to the results obtained in the data-limited paradigm, which suggest that word units reveal their component letters, the results from the resource-limited paradigm imply that activated word units conceal their component letters. If familiarity effects are due to the different units used when displays are processed, then the conditions that determine the relationship between a whole display and its components should in turn modulate the familiarity effect. The present experiments tested the unitization hypothesis, under resource-limited conditions characterized by relatively long exposure durations and the absence of a postdisplay mask. The chief reasons for making that choice were reservations raised about the procedures used to control the level of performance in the datalimited paradigm (Marchetti \& Mewhort, 1986), as well as a recent study of unitization (Silverman, 1985) that provided the empirical contrast for the present studies.

Silverman (1985) examined whether perceptual units intermediate in size relative to single-letter and wholeword units were functional in a matching task. In the matching task, a target item and comparison display are presented, and the subject decides whether the target is contained in the comparison display. Silverman criticized previous studies that addressed the issue of perceptual units with this task (Santa, Santa, \& Smith, 1977), on the assumption that lateral presentation of the target and comparison items may have led to differential positional uncertainty among targets of varying sizes, and that those studies did not compare word and nonword processing. Silverman presented targets that varied in the number of constituent letters directly above the critical letters in the comparison displays, which were words or nonwords.

Three criteria were used by Silverman (1985) to index perceptual unitization of multiletter targets: (1) a word familiarity effect should be found; (2) multiletter targets should be matched as quickly as single-letter targets when the comparison display formed a word; and (3) singleletter targets should be matched more quickly than multi- 
letter targets when the comparison display formed a nonword. The only multiletter target that satisfied all the criteria was the entire four-letter word display. Although other targets did display differences between words and nonwords, those familiarity effects were attributed to nonperceptual processes. The conclusion that a whole display functions as a unit for words but not for nonwords is consistent with the unitization hypothesis. However, Silverman's results regarding the second criterion above are not consistent with findings reported by Marmurek (1977). Specifically, Marmurek found letterin-word matches to be faster than whole-word matches under conditions of simultaneous presentation, an effect that has been replicated by Umansky and Chambers (1980). These findings suggest that the familiarity effect for whole words would not satisfy the criteria proposed by Silverman (1985) to index perceptual unitization.

Experiment 1 was designed to determine whether the different relative speeds of whole-word and letter-in-word decisions were due to the testing of all positions by Silverman (1985) and the testing of only the firstletter position by Marmurek (1977) and Umansky and Chambers (1980). It may be that the potential positional uncertainty effects noted by Silverman in the Santa et al. (1977) study also apply to Silverman's study. That is, in a block of trials in which the subjects knew that a singleletter target was to be compared, the subjects did not know in which position the target would appear. This uncertainty might have led the subjects to locate and switch attention to the critical position of the target. Either process may have been carried out more quickly for words than for nonwords. Furthermore, the absence of an attentionswitching process for whole-word comparisons would lend a processing time advantage to whole-word comparisons relative to single-letter comparisons (LaBerge, 1983; Marmurek, 1987). That advantage would nullify the singleletter processing advantage when positional uncertainty is removed (Marmurek, 1977; Umansky \& Chambers, 1980).

Experiment 1 tested the prediction that single-letter matches should be made more quickly than whole-word matches when the switching process is eliminated from the single-letter condition. To examine the effects of positional uncertainty on the familiarity effect, the single-letter conditions studied by Silverman (1985) were contrasted with single-letter conditions in which subjects were informed about the location of the target letter. A wholedisplay comparison condition was included to determine the dependency of the familiarity effect on the part-whole relationship between the display and its component letters.

\section{EXPERIMENT 1}

\section{Method}

Subjects and Design. The subjects were 24 introductory psychology students at the University of Guelph. All subjects reported having normal or corrected-to-normal vision and were native readers of English. Each subject served in all 36 conditions of the experiment. Those conditions were formed by the orthogonal combina- tion of three factors. The type of display factor contrasted words and nonwords. The response factor contrasted matches and mismatches between the target and comparison items. The third factor was type of comparison, and there were nine levels. Four levels tested single-letter targets in a constant position for a block of trials, with each of four positions tested equally often. Four levels tested single-letter targets in mixed blocks with each position tested equally often, but in a random order, within a block. The final condition tested all four positions simultaneously.

Stimuli. Two types of stimuli were selected in accordance with the procedures followed by Silverman (1985). Four-letter words designated A or AA in the Thorndike and Lorge (1944) norms were used along with nonwords formed by permuting the letters of the words. Words and nonwords were paired so that the target letter was matched for a pair. The words and nonwords were intermixed among the trials. Silverman (1985) showed that blocking by display type and mixing the two types of displays did not yield different results.

Each subject was tested on 240 experimental trials. In the singleletter fixed position conditions, 96 items were used such that each position was tested six times in four conditions: word match, nonword match, word mismatch, and nonword mismatch. Similarly, there were 96 trials in single-letter mixed position conditions. There were 48 whole-display trials with 12 instances each for word match, nonword match, word mismatch, and nonword mismatch. No word or nonword was presented twice to any subject. On the 24 mismatch trials, only one letter differed between the two displays, and each position was tested equally often.

Procedure. In the single-letter target conditions, the subjects were tested in blocks of 24 trials. In the four fixed blocks, the position of the target letter was held constant, and the subjects were informed of the critical position prior to the block. The order of these four blocks was counterbalanced across subjects. The blocks of trials in the mixed conditions tested each position equally often in a random order. There were four of those blocks tested in a counterbalanced order. In the whole-display conditions, there were two blocks of 24 trials, and the order of blocks was counterbalanced across subjects. The blocks for each of the major conditions (fixed, mixed, whole) were themselves blocked, and the order of the three blocks was counterbalanced across subjects.

All displays were shown centered on an Electrohome monitor connected to a GIMLX microcomputer. The letters in the displays were shown in uppercase and were approximately $8 \mathrm{~mm}$ in height and $4 \mathrm{~mm}$ in width. The subjects viewed the displays from a distance of approximately $60 \mathrm{~mm}$. Targets were aligned directly above the comparison items. For single-letter trials, the subjects judged whether the letter matched the letter directly below it. On wholedisplay trials, all letters had to match. Two response keys marked "yes" and "no"' were connected to the microcomputer, and the subjects selected which hand was assigned to each key. The index finger of the hand was used to indicate responses. The latency and accuracy of responses were stored by the computer.

Prior to each block of trials, the subjects received 12 practice trials. All trials began with the presentation of the word READY in the center of the screen for $500 \mathrm{msec}$. After a blank delay of $500 \mathrm{msec}$, the target and comparison item were shown simultaneously until the response was made or until $3,000 \mathrm{msec}$ had elapsed. There was a delay of about $2 \mathrm{~min}$ between blocks during which the experimenter called up the appropriate program. No feedback was given to the subjects until all conditions had been completed.

\section{Results and Discussion}

Because different processes precede the execution of a "yes" and a "no" response (Farell, 1985), and in order to make direct comparisons with Silverman (1985), separate analyses were run on the two types of response. The 
mean correct decision latencies and the mean error rates for "yes" and "no" trials are shown in Table 1 and Table 2 , respectively. The overall correlation between mean correct latencies and mean error rates was $r(34)=.56$, $p<.001$. That is, there was no speed-accuracy tradeoff; rather, difficult conditions led to longer latencies and higher error rates.

"Yes" trials. A 2 (word vs. nonword) $\times 9$ (comparison condition) completely within-subjects ANOVA run on the mean error rates on "yes"' trials showed no significant effects. A similar analysis on the mean correct latencies showed that the main effect of comparison condition was significant $\left[F(8,184)=10.87, M S_{\mathrm{e}}=20,459\right.$, $p<.001]$, as was the display $\times$ comparison interaction $\left[F(8,184)=3.68, M S_{\mathrm{e}}=13,642, p<.001\right]$. The interaction was probed in order to determine which comparison conditions differed within each display level and to determine which comparison conditions yielded familiarity effects.

For words, the four single-letter fixed conditions did not differ significantly $[F(3,184)=1.23]$, and the four singleletter mixed conditions did not differ $[F(3,184)<1]$. Accordingly, the two sets of single-letter conditions were pooled across position. A separate analysis compared the mean pooled latency for the fixed condition $(M=$ $717 \mathrm{msec})$, the mean pooled latency for the mixed condition $(M=787 \mathrm{msec})$, and the mean latency for the whole-display condition $(M=806 \mathrm{msec})$. The means differed significantly $\left[F(2,46)=10.55, M S_{\mathrm{e}}=7,820\right.$, $p<.001]$. Linear contrasts showed that the mean latency in the fixed condition was shorter than in the mixed condition $[F(1,46)=15.04, p<.001]$, and that the mixed and whole-word latencies did not differ $[F(1,46)=2.83$, $p>$.05].

These results replicate the findings by Silverman (1985) that when single-letter comparisons are tested randomly across positions, they are made no more quickly than whole-word decisions. However, whole-word decisions are not made as quickly as single-letter decisions when the position of the letter is known in advance. The latter result suggests that single-letter matching is impaired when

Table 1

Mean Correct Latencies (in msec) and Error Rates (\%) on "Yes" Trials: Experiment 1

\begin{tabular}{|c|c|c|c|c|}
\hline \multirow[b]{3}{*}{ Condition } & \multicolumn{4}{|c|}{ Display } \\
\hline & \multicolumn{2}{|c|}{ Word } & \multicolumn{2}{|c|}{ Nonword } \\
\hline & $M$ & Error Rate & $M$ & Error Rate \\
\hline \multicolumn{5}{|l|}{ Fixed-letter } \\
\hline First & 694 & 1.50 & 710 & 1.33 \\
\hline Second & 713 & 1.33 & 738 & 1.33 \\
\hline Third & 738 & 0.17 & 744 & 2.67 \\
\hline Fourth & 723 & 1.33 & 718 & 2.67 \\
\hline \multicolumn{5}{|l|}{ Mixed-letter } \\
\hline First & 775 & 2.67 & 801 & 3.83 \\
\hline Second & 794 & 1.50 & 815 & 4.00 \\
\hline Third & 785 & 1.33 & 771 & 2.67 \\
\hline Fourth & 795 & 1.50 & 775 & 1.33 \\
\hline Whole-display & 806 & 1.33 & 1,068 & 2.67 \\
\hline
\end{tabular}

Table 2

Mean Correct Latencies (in msec) and Error Rates (\%) on "No" Trials: Experiment 1

\begin{tabular}{|c|c|c|c|c|}
\hline \multirow[b]{3}{*}{ Condition } & \multicolumn{4}{|c|}{ Display } \\
\hline & \multicolumn{2}{|c|}{ Word } & \multicolumn{2}{|c|}{ Nonword } \\
\hline & $M$ & Error Rate & $M$ & Error Rate \\
\hline \multicolumn{5}{|l|}{ Fixed-letter } \\
\hline First & 782 & 3.33 & 763 & 0.67 \\
\hline Second & 853 & 4.33 & 801 & 2.67 \\
\hline Third & 804 & 3.67 & 795 & 0.67 \\
\hline Fourth & 788 & 3.67 & 794 & 1.67 \\
\hline \multicolumn{5}{|l|}{ Mixed-letter } \\
\hline First & 840 & 2.33 & 857 & 1.33 \\
\hline Second & 887 & 2.67 & 872 & 3.33 \\
\hline Third & 877 & 1.33 & 889 & 3.33 \\
\hline Fourth & 843 & 0.67 & 870 & 3.33 \\
\hline Whole-display & 968 & 5.33 & 1,103 & 5.67 \\
\hline
\end{tabular}

subjects must switch attention to a target location after a display is presented, a process precluded by the blocking of trials by target position. When trials are blocked by position, there is no evidence that words are treated as perceptual units according to the criteria proposed by Silverman.

For nonwords, the four single-letter fixed conditions did not differ significantly $[F(3,184)<1]$, nor did the four single-letter mixed conditions $[F(3,184)=1.16]$. Accordingly, the two sets of single-letter conditions were pooled across position. A separate analysis compared the mean pooled latency for the fixed condition $(M=$ $728 \mathrm{msec}$ ), the mean pooled latency for the mixed condition $(M=791 \mathrm{msec})$, and the mean latency for the whole-display condition $(M=1,068 \mathrm{msec})$. The means differed significantly $\left[F(2,46)=28.27, M S_{\mathrm{e}}=19,216\right.$, $p<.001]$. Linear contrasts showed that the fixed condition did not differ from the mixed condition $[F(1,46)=$ $2.48, p>.05]$, but that both single-letter conditions $(M=760 \mathrm{msec})$ led to shorter latencies than did the whole-nonword condition $[F(1,46)=52.25, p<.001]$. These nonword results replicate the single-letter advantage over whole comparisons found by Silverman (1985). The effect indicates that nonwords are not treated as perceptual units.

To test for familiarity effects, the word and nonword means were compared in each of the three major conditions. Word and nonword latencies did not differ in either the fixed or mixed single-letter conditions $[F(1,23)<1$ in both cases]. However, whole-word comparisons were significantly faster than whole-nonword comparisons $[F(1,23)=58.43, p<.001]$. Both sets of outcomes replicate Silverman (1985), but suggest that the familiarity effect is not due to perceptual unitization, because words fail the criterion of being matched as quickly as single letters when the position of the letter is fixed.

"No" trials. The 2 (word vs. nonword) $\times 9$ (comparison condition) ANOVAs run on the error rates and correct latencies on "no" trials showed that the only significant main effect was for comparison condition in the latency analysis $\left[F(8,184)=24.45, M S_{\mathrm{e}}=12,980\right.$, 
$p<.001]$. The fixed-position latencies $(M=797 \mathrm{msec})$ were faster than the mixed-position latencies $[M=$ $867 \mathrm{msec} ; F(1,184)=4.25$ ], and the mixed-position latencies were faster than the whole-display latencies $[M=1,035 ; F(1,184)=29.13]$. The analyses show that the pattern of significant effects found for "yes" trials did not appear on "no" trials (Farell, 1985). This may in part be due to time-consuming rechecking processes, which are more likely to occur on "no" trials $(M=855 \mathrm{msec})$ than on "yes" trials $(M=775 \mathrm{msec})$.

Because Silverman (1985) reported a significant difference between word and nonword latencies on "no" trials, a planned comparison was carried out on the present data even though the display $\times$ comparison interaction was not statistically significant. The error term was that used to test the interaction effect, and the comparison showed that the 97 -msec difference was significant $[F(1,184)=$ 4.91, $M S_{\mathrm{e}}=22,989$ ]. This is another instance of a familiarity effect that is not due to perceptual unitization, as is indicated by the faster latencies to letter-in-word than whole-word comparisons.

\section{EXPERIMENT 2}

The major conclusion from Experiment 1 was that the data interpreted by Silverman (1985) to be consistent with a perceptual unitization account of familiarity effects were an artifact of differential attentional demands in the singleletter and whole-word tasks. LaBerge (1983) has shown that attention is more narrowly focused for single-letter than for whole-word decisions. The use by Silverman of a random ordering of target letter positions in single-letter blocks imposed on subjects the task of determining where to focus attention when locating the target. This locational uncertainty slowed down performance relative to the fixed-location conditions, under which there was no evidence for word unitization; that is, fixed-letter latencies were shorter than whole-word latencies. According to the criteria proposed by Silverman, that pattern of results indicates that the familiarity effects found in Experiment 1, and by Silverman, were not due to the perceptual unitization of a word.

If the familiarity effect in the simultaneous visual comparison task is not due to perceptual unitization, to what is it due? Part of the problem in addressing this issue concerns the definition of "perceptual unitization." Whereas Henderson (1982) claimed that "perception has been construed in terms of the uptake of information" (p. 288), Silverman (1985) suggested that unitization effects emerge from the matching process. The evidence testing between input coding and comparison as loci of familiarity effects was reviewed by Krueger (1975). The data from the visual comparison studies available then (notably, there was no analysis of whole vs. part visual comparison tasks) were consistent with the view that both processes contribute to the familiarity effect.
One purpose for Experiment 2 was to examine the contribution of encoding processes to the familiarity effect. Proctor (1981) has argued that sequential presentation, relative to simultaneous presentation, facilitates the encoding of the comparison item. If familiarity effects arise during encoding, then the magnitude of those effects might be reduced in the sequential presentation condition where encoding is facilitated.

This reduction in the familiarity effect would be expected if encoding facilitation in the sequential task is especially effective for unfamiliar items. That is, subjects might form a cognitive code (Proctor, 1981) during the interitem interval, which could be used to guide the encoding of the comparison item. That holistic codes are used in a sequential task is indicated by faster whole-display than fixed-letter decisions (Johnson, 1975; LaBerge, 1983; Marmurek, 1977), a result that contrasts with the slower whole-word decisions in Experiment 1. Other evidence for whole-unit processing in a sequential task is the absence of an effect of word length on matching times (Johnson, 1975), an effect that is present in simultaneous matching (Eichelman, 1970). A comparison of the magnitudes of the familiarity effects in the simultaneous and sequential matching tasks in Experiment 2 provided an index of the extent to which familiarity effects depend upon the activation of holistic units in memory to guide the encoding of the comparison item.

\section{Method}

Subjects and Design. The subjects were 48 introductory psychology students whose participation fulfilled a course requirement. Two groups were formed by assigning subjects in a block randomized order to the sequential or simultaneous task. The subjects within each group participated in the 36 conditions tested in the simultaneous task used in Experiment 1.

Stimuli and Procedure. The blocking schedules and apparatus were identical to those used in Experiment 1, as were the timing parameters for the simultaneous condition. In the sequential condition, the target was shown for $500 \mathrm{msec}$ and was followed by a blank delay of $1,000 \mathrm{msec}$. The comparison item was then shown until the response was made, or until $3,000 \mathrm{msec}$ had elapsed.

The format of the single-letter trials was different than in Experiment 1 , in which the location of the target position was specified by means of presenting the letter above the critical comparison item letter. This procedure was not possible in the sequential condition. Therefore, the target letter was accompanied by dashes (hyphens) in the noncritical positions. This format was used in both the simultaneous and sequential conditions of Experiment 2, to ensure that differences between the simultaneous and sequential conditions were not attributable to different formats of presentation.

\section{Results and Discussion}

Preliminary analyses showed that, as in Experiment 1, the position of the letter on single-letter trials did not affect error rates or correct latencies. Accordingly, the data were pooled across position. The "yes" data for the 2 (task: simultaneous vs. sequential) $\times 2$ (display: word vs. nonword) $\times 3$ (comparison: fixed-letter; mixed-letter; whole-display) mixed design with task as the only between-subjects factor are shown in Table 3. The "no" 
Table 3

Mean Correct Latencies (in msec) and Error Rates (\%) on "Yes" Trials: Experiment 2

\begin{tabular}{|c|c|c|c|c|c|}
\hline \multirow[b]{3}{*}{ Task } & \multirow[b]{3}{*}{ Condition } & \multicolumn{4}{|c|}{ Display } \\
\hline & & \multicolumn{2}{|r|}{ Word } & \multicolumn{2}{|c|}{ Nonword } \\
\hline & & $M$ & Error Rate & $M$ & Error Rate \\
\hline Simultaneous & Fixed-letter & 713 & 1.33 & 719 & 2.33 \\
\hline Sirnultaneous & Mixed-letter & 785 & 1.83 & 800 & 2.67 \\
\hline Simultaneous & Whole-display & 830 & 1.33 & 1,010 & 3.33 \\
\hline Sequential & Fixed-letter & 667 & 2.67 & 643 & 4.67 \\
\hline Sequential & Mixed-letter & 683 & 2.67 & 704 & 3.33 \\
\hline Sequential & Whole-display & 616 & 0.67 & 702 & 3.67 \\
\hline
\end{tabular}

Table 4

Mean Correct Latencies (in msec) and Error Rates (\%) on "No" Trials: Experiment 2

\begin{tabular}{|c|c|c|c|c|c|}
\hline \multirow[b]{3}{*}{ Task } & \multirow[b]{3}{*}{ Condition } & \multicolumn{4}{|c|}{ Display } \\
\hline & & \multicolumn{2}{|r|}{ Word } & \multicolumn{2}{|c|}{ Nonword } \\
\hline & & $M$ & Error Rate & $M$ & Error Rate \\
\hline Simultaneous & Fixed-letter & 796 & 3.33 & 781 & 1.67 \\
\hline Simultaneous & Mixed-letter & 851 & 1.83 & 859 & 2.83 \\
\hline Simultaneous & Whole-display & 985 & 4.75 & 1,082 & 5.63 \\
\hline Sequential & Fixed-letter & 731 & 3.67 & 740 & 2.33 \\
\hline Sequential & Mixed-letter & 758 & 1.67 & 756 & 3.33 \\
\hline Sequential & Whole-display & 767 & 4.67 & 774 & 5.67 \\
\hline
\end{tabular}

data are shown in Table 4. The overall correlation between error rates and correct latency was $r(22)=.33$, $p>.05$.

"Yes" trials. A 2 (task) $\times 2$ (display) $\times 3$ (comparison) mixed ANOVA of the error data showed that the percentage of errors was higher for nonwords $(M=3.33)$ than for words $\left[M=1.75 ; F(1,46)=47.29, M S_{\mathrm{e}}=\right.$ $7.33, p<.001]$. However, the task $\times$ display interaction was significant $[F(1,46)=10.38, p<.01]$, and showed that the difference in error rates was more pronounced in the sequential task. No other effects were significant in the analysis of error rates.

A 2 (task) $\times 2$ (display) $\times 3$ (comparison) mixed ANOVA of the correct "yes" latencies showed that all main effects and interactions were statistically significant. For the task $\times$ display $\times$ comparison interaction $[F(2,92)=3.59, p<.05]$. Separate 2 (display) $\times 3$ (comparison) ANOVAs were run on each group of subjects to examine how the task variable influenced the display $\times$ comparison interaction. In the simultaneous group, latencies were faster for words $(M=776 \mathrm{msec})$ than for non-words $\left[M=843 \mathrm{msec} ; F(1,23)=29.37, M S_{\mathrm{e}}=\right.$ $5,559, p<.001]$, and the latencies differed across the fixed $(M=716 \mathrm{msec})$, mixed $(M=793 \mathrm{msec})$, and whole $(M=920 \mathrm{msec})$ comparison conditions $[F(2,46)=$ $\left.26.33, M S_{\mathrm{e}}=19,352, p<.001\right]$. The display $\times$ comparison interaction was also significant $[F(2,46)=15.13$, $M S_{\mathrm{e}}=7,681, p<.001$ ]. Linear contrasts (summarized in the top portion of Table 5) showed that, for words, the fixed-letter latencies were shorter than the mixed-letter and the whole-word latencies. The latter two conditions did not differ. For nonwords, fixed-letter comparisons were faster than mixed-letter comparisons, and the latter were faster than whole comparisons.

Contrasts between words and nonwords in each of the comparison conditions showed that latencies differed only in the whole-display condition $[F(1,46)=50.61]$. These results essentially replicate three critical findings of Experiment 1: that positional uncertainty slows single-letter decisions; that whole words are compared more slowly than are a letter target and a letter in a fixed position in a word; and, that the familiarity effect occurs only for whole-display comparisons. The second finding, following Silverman (1985), rules out the interpretation that the familiarity effect is due to perceptual unitization.

In the sequential task, latencies were faster for words $(M=655 \mathrm{msec})$ than for nonwords $[M=683 \mathrm{msec}$; $\left.F(1,23)=5.42, M S_{\mathrm{e}}=5,225, p<.05\right]$, and latencies in the fixed $(M=655 \mathrm{msec})$, mixed $(M=694 \mathrm{msec})$, and whole $(M=659 \mathrm{msec})$ conditions differed significantly $[F(2,46)=4.19, p<.05]$. The display $\times$ comparison interaction was significant $[F(2,46)=16.61$, $\left.M S_{\mathrm{e}}=2,203, p<.001\right]$. Linear contrasts (summarized in the bottom portion of Table 5) showed that, for words, the two letter target conditions did not differ, and both types of single-letter decisions were made more slowly than whole-word comparisons. For nonwords, fixed-letter decisions were made more quickly than mixed-letter decisions; however, the mixed-letter and whole comparisons did not differ.

Comparisons between the two types of displays showed that the trend towards a familiarity disadvantage in the fixed-letter conditions was not significant $[F(1,46)=$ $3.13]$. The familiarity effect in the whole-display condition was significant $[F(1,46)=40.29]$.

The pattern for word decisions in the two tasks is similar to that found by Marmurek (1977). That is, whereas the simultaneous group showed faster decisions for fixedletter than for whole-word comparisons, the sequential group showed faster whole-word comparisons than fixedletter comparisons. These results are consistent with the view that word units are more likely to dominate processing in the sequential task than in the simultaneous task. To determine directly whether word-unit activation influences the familiarity effect, the familiarity effect was contrasted between the two groups. The difference be-

Table 5

Summary of the Linear Contrasts for the Task $\times$ Display $\times$ Comparison Interaction

\begin{tabular}{lllrc}
\hline \multicolumn{1}{c}{ Task } & Condition & \multicolumn{1}{c}{ Contrast } & $F(1,46)$ & $p$ \\
\hline Simultaneous & Words & Fixed- vs. Mixed-letter & 7.88 & $*$ \\
Simultaneous & Words & Mixed-letter vs. Whole & 3.31 & n.s. \\
Simultaneous & Nonwords & Fixed- vs. Mixed-letter & 10.25 & $*$ \\
Simultaneous & Nonwords & Mixed-letter vs. Whole & 68.89 & $*$ \\
Sequential & Words & First- vs. Mixed-letter & 1.31 & n.s. \\
Sequential & Words & Mixed-letter vs. Whole & 25.28 & $*$ \\
Sequential & Nonwords & Fixed- vs. Mixed-letter & 20.26 & $*$ \\
Sequential & Nonwords & Mixed-letter vs. Whole & 0.89 & n.s. \\
\hline
\end{tabular}

*Alpha set at .01 . 
tween whole-nonword correct latency and whole-word correct latency was calculated for each subject, and the differences between the two groups were compared. The familiarity effect was larger in the simultaneous condition $(M=180 \mathrm{msec})$ than in the sequential condition $[M=$ $\left.86 \mathrm{msec} ; F(1,46)=8.74, M S_{\mathrm{e}}=11,406, p<.01\right]$. This result suggests that the slower encoding processes in the simultaneous task contribute significantly to the familiarity effect.

"No" trials. A 2 (task) $\times 2$ (display) $\times 3$ (comparison) mixed ANOVA of the error rates showed that no main effects or interactions were significant (all $p \mathrm{~s}>.05$ ). A similar ANOVA for the mean correct latencies showed that latencies were longer in the simultaneous task $(M=$ $892 \mathrm{msec}$ ) than in the sequential task $[M=754 \mathrm{msec}$; $\left.F(1,46)=16.06, M S_{\mathrm{e}}=84,840, p<.001\right]$, and that latencies were longer for nonwords $(M=832 \mathrm{msec})$ than for words $[M=815 \mathrm{msec}) ; F(1,46)=4.63, M S_{\mathrm{e}}=$ $4,730, p<.05]$. More importantly, the display factor did not enter into any interactions. That is, the familiarity effect was not affected by the task or the type of comparison on "no" trials. However, because Silverman (1985) found a significant familiarity effect on simultaneous "no" trials, which was also obtained in Experiment 1 , a contrast was carried out, and the effect was found to be significant $[F(1,92)=10.43, p<.001]$.

The comparison main effect was significant $[F(2,92)=$ $\left.38.80, M S_{\mathrm{e}}=12,632, p<.001\right]$, and that factor entered into a significant interaction with task $[F(2,92)=23.14$, $p<.001]$. Linear contrasts for the simultaneous group showed that the fixed-letter latencies were shorter than the mixed-letter latencies $[F(1,92)=4.14, p<.05]$, and that the mixed-letter latencies were shorter than the wholedisplay latencies $[F(1,92)=30.09, p<.001]$. These results replicate those of Experiment 1 and Silverman's (1985). Although the direction of differences among the comparisons was similar on the sequential task, none of the contrasts was significant.

\section{GENERAL DISCUSSION}

Both experiments showed that a whole word is matched more slowly than a fixed-letter target in a simultaneous matching task. That result is inconsistent with the criterion proposed by Silverman (1985) to index perceptual unitization of a word as the basis of familiarity effects. The apparent similarity of processing whole words and a letter within a word reported by Silverman was due to the increased time required to determine the location of the target letter when its location was varied randomly within a block of trials.

Silverman (1985) attributed the familiarity effect for whole displays to the greater likelihood of using a holistic comparison process for words than for nonwords. Experiment 2, however, showed that the familiarity effect was larger in the simultaneous matching task than in the sequential matching task. If sequential presentation leads to facilitation in the encoding of a repeated item (Proctor,
1981), then the task $\times$ display interaction indicates that nonwords benefit more (difference $=308 \mathrm{msec}$ ) during encoding than do words (difference $=214 \mathrm{msec}$ ). That is, during the interitem interval, subjects may establish a cognitive code for the nonword target that could be used in encoding the nonword comparison item. In the simultaneous task, that code would not be generated; hence, the larger familiarity effect on simultaneous trials.

The absence of familiarity effects on single-letter target trials is consistent with the view that the familiarity effect arises from the facilitation in processing due to the activation of the word's cognitive code. When a letter is predesignated as the target, higher order cognitive codes are not activated to prime the processing of the word comparison item (Johnston \& McClelland, 1974). For both types of displays, the effects of target position are similar in the simultaneous and sequential tasks, providing further evidence that processing is equivalent for both types of displays when the targets are parts of a display.

The present account of the different familiarity effects in the simultaneous and sequential tasks does not assume that the encoding of two items is strictly serial in the simultaneous task. It is only assumed that the completion of the encoding of the target precedes the completion of the encoding of the other item. The completed encoding may then guide the encoding of the not yet completely encoded item. It is possible that when two items are presented together, subjects attempt to encode both items simultaneously (McClelland \& Mozer, 1986). It is likely, however, that simultaneous encodings will be maximized by uncertainty as to which item is to be tested as a target, as in the McClelland and Mozer studies. In the present studies, as in reading, the relative positions of the target and context items were fixed, a condition that leads subjects to focus attention on single words (McConkie \& Zola, 1987).

The original empirical aim of the present studies was to examine the limiting conditions giving rise to faster word than letter-in-word comparisons in a simultaneous matching task (Silverman, 1985). It was shown that the outcome is due to the positional uncertainty of single-letter targets. When that uncertainty is removed, words are matched more slowly than their component letters. This is not to indicate, however, that multiletter units are not functional in word perception. In the sequential task, whole words are matched more quickly than their component letters. Moreover, words can prime the identification of subsequently presented component bigrams (Greenberg \& Vellutino, 1988). The sequential task, then, reveals that multiletter units are activated by the prior encoding of a word.

In conclusion, familiarity effects occur when cognitive units available for words are activated (cf. Healy \& Drewnowski, 1983; Marmurek, 1987). One result of that activation is the facilitation afforded the encoding of the word comparison items. Such facilitation by cognitive units is not available for the nonwords, leading to an increase in the number of codings compared for nonwords 
relative to words. Given a delay between the target and comparison item, cognitive units of nonwords may be established to reduce both the encoding and comparison differences between words and nonwords. The residual familiarity effect in the sequential task may indicate the lower probability of successfully generating a cognitive code for nonwords.

\section{REFERENCES}

Eichelman, W. (1970). Familiarity effects in the simultaneous matching task. Journal of Experimental Psychology, 26, 566-581.

FARELL, B. (1985). "Same"-" "different" judgments: A review of current controversies in perceptual comparisons. Psychological Bulletin, 98, 419-456.

GreEnBerg, S. N., \& Vellutino, F. R. (1988). Evidence for processing of constituent single- and multiletter codes: Support for multilevel coding in word perception. Memory \& Cognition, 16, 54-63.

Healy, A. F., \& Drewnowskı, A. (1983). Investigating the boundaries of reading units: Letter detection in misspelled words. Joumal of Experimental Psychology: Human Perception \& Performance, 9, 413-426.

HeNDERSON, L. (1982). Orthography and word recognition in reading. London: Academic Press.

JoHNSON, N. F. (1975). On the function of letters in word identification: Some data and a preliminary model. Journal of Verbal Learning \& Verbal Behavior, 14, 17-29.

Johnston, J. C., \& McClelland, J. L. (1974). Perception of letters in words: Seek not and ye shall find. Science, 184, 1192-1194.

KRUEGER, L. E. (1975). Familiarity effects in visual information processing. Psychological Bulletin, 82, 949-974.

LABERGE, D. (1983). Spatial extent of attention to letters and words.
Journal of Experimental Psychology: Human Perception \& Performance, 15, 371-379.

Marchetti, F. M., \& Mewhort, D. J. K. (1986). On the wordsuperiority effect. Psychological Research, 48, 23-35.

Marmurek, H. H. C. (1977). Processing letters in words at different levels. Memory \& Cognition, 5, 67-72.

Marmurex, H. H. C. (1987). Attentional holism in visual word processing. Psychological Research, 49, 45-52.

MCClelland, J. L., \& Mozer, M. C. (1986). Perceptual interactions in two-word displays: Familiarity and similarity effects. Journal of Experimental Psychology: Human Perception \& Performance, 12, 18-35.

McConkie, G. W., \& Zola, D. (1987). Visual attention during eye fixations while reading. In M. Coltheart (Ed.), Attention and performance XII: The psychology of reading (pp. 385-402). East Sussex, England: Erlbaum.

Proctor, R. W. (1981). A unified theory for matching-task phenomena. Psychological Review, 88, 291-326.

Santa, J. L., Santa, C., \& Smith, E. E. (1977). Units of word recognition: Evidence for the use of multiple units. Perception \& Psychophysics, 22, 585-591.

SANTEe, J. L., \& Egeth, H. E. (1982). Do reaction time and accuracy measure the same aspects of letter recognition? Journal of Experimental Psychology: Human Perception \& Performance, 8, 489-501.

Silverman, W. P. (1985). Two types of word superiority effects in a speeded matching task. Memory \& Cognition, 13, 50-56.

THORNDIKE, E. \& LORGE, I. (1944). The teacher's word book of 30,000 words. New York: Teachers College Press, Columbia University.

Umansky, J. A., \& Chambers, S. M. (1980). Letters and words in word identification. Memory \& Cognition, 8, 433-446.

(Manuscript received June 8, 1988; revision accepted for publication December 16, 1988.) 